PREVENTIVE HEALTHCARE:

TOPICAL ISSUES OF HEALTH RISK ANALYSIS

UDC 613.956

DOI: 10.21668/health.risk/2019.2.01.eng

\title{
BASIC TRENDS IN BEHAVIORAL HEALTH RISKS
}

\section{V.R. Kuchma, S.B. Sokolova}

National Medical Research Center for Children's Health, 1 Bldg., 2 Lomonosov Avenue, Moscow, 119991, Russian Federation

A scope of research entitled Health Behaviour in School-Aged Children [HBSC] allowed to obtained data for further analysis and systematization; there were some basic trends in behavioral risks revealed as a result which were hazardous for health of schoolchildren in Russia aged 11, 13, and 15. We applied data taken from reports on international HBSC research conducted in 1993, 1997, 2001, 2005, 2009, and 2013. All the data were statistically processed with nonparametric analysis. We compared risk factors prevalence among children aged 11, 13, and 15; among boys and girls; over time dynamics; factors revealed in different countries. We detected both age and gender peculiarities in behavioral factors that influenced health; there were also discrepancies in their prevalence taken in dynamics over years and differences between children of the same age living in Russia and abroad. A lot of risky behavioral patterns are more widely spread among boys. Since 1993 there has been a growth in parameters related to risky behaviors among girls in Russia. Over the last ten years there have been positive trends in behavioral risk factors prevalence. However, when compared with their foreign counterparts, Russian teenagers perceive their school environment more negatively; they more frequently tend to estimate their health as "being poor"; they are less satisfied with their lives; they less frequently consume fruit and pay less attention to oral health; they tend to be physically inactive. Risky behavioral patterns such as smoking, alcohol use, or aggressive behaviour, are more widely spread among younger teenagers. When creating programs aimed at establishing healthy lifestyle, it is necessary to take into account age and gender differences, as well as trends related to behavioral risk factors which change over time and which can be objectively assessed with an international questionnaire entitled Health Behaviour in School-Aged Children.

Key words: children and teenagers hygiene, behavioral risk, non-parametric analysis, time dynamics, gender difference, healthy lifestyle, international questionnaire.

\section{Introduction}

Health of coming generations still tends to deteriorate and requires immediate attention given to it by the overall society [1-7].

Health disorders occur due to risk factors that lead to greater probability of various diseases, their development, and adverse outcomes [8-9].

According to contemporary concepts, a person's health is a result of complicated convergence that comprises biological (physiological and genetic), behavioral, social, economic, and ecological factors. WHO experts spot out four groups of factors that determine one's health; they are heredity (20\%), environmental factors $(20 \%)$, quality and availability of medical aid $(10 \%)$, and factors related to a person's lifestyle (50\%). An extent to which various factors can influence functional state of a schoolchild's body depends on his or her age; thus, contribution made into it by physical activity amounts to $45.3 \%$ for junior schoolchildren (aged 7-11), and to $18.4 \%$, for older ones (aged 12-18); social factors, to $19.7 \%$ and $9.6 \%$ respectively; educational loads, $10.2 \%$ and $35.8 \%$ : lifestyle factors, $9.8 \%$ and

(c) Kuchma V.R., Sokolova S.B., 2019

Vladislav R. Kuchma - Corresponding Member of the RAS, Doctor of Medical Sciences, Professor, Deputy Director responsible for research, Head of the Scientific Research Institute for Children Health Protection and Hygiene "National Medical Research Center for Children Health" of the Russian Federation Public Healthcare Ministry (e-mail: info@niigd.ru; tel.: +7 (495) 917-48-31; ORCID: http://orcid.org/0000-0002-1410-5546).

Svetlana B. Sokolova - Candidate of Medical Sciences, Leading researcher (e-mail: s-s-b@mail.ru; tel.: +7 (495) 917-48-31; ORCID: https://orcid.org/0000-0001-5895-4577). 
$27.6 \%$; learning conditions, $15.0 \%$ and $8.6 \%$, respectively [10].

Lifestyle-related adverse factors tend to prevail significantly among contemporary schoolchildren and in future it is these factors that will determine mortality and morbidity (their levels and dynamics) among adult population [11-17].

As per data provided by $\mathrm{WHO}, 70 \%$ untimely death cases among adults are related to behavioral factors that first occurred during teenage years. Children's attitudes towards their own health tend to differ greatly from those of adults'. WHO experts state there are about 60 behavioral factors that influence teenagers' health [18]. International research entitled "Health Behaviour in School-Aged Children [HBSC]" enables obtaining the most comprehensive picture of teenagers' health and well-being. Research within HBSC project is accomplished once in four years. Its tool is a standard questionnaire developed by an international scientific-research network and its target groups are schoolchildren aged 11, 13, and 15. A minimal recommended sampling is equal to 1,500 schoolchildren for each age group. Questioning is usually performed by specially trained teachers, school nurses, and experts from scientific research institutes. Most countries and regions stratify their samplings in order to provide as wide geographic coverage as it's only possible.

HBSC project was first introduced in 1983 in five countries, and soon its participants established relations with WHO European Office. At present there are 44 countries in Europe and North America that participate in it. The project network includes 340 researchers who are employed at public healthcare universities and institutes. Since 1993, this research has been accomplished in Russia according to a unified procedure that allowed comparing results obtained for different groups of children as well as in different years.

A range of project priorities is constantly being enlarged as per their contents and aspects related to exploring health and behavioral issues as regards schoolchildren. First research works tended to focus on such factors as smoking, physical activity and certain psychosocial health aspects. Later on some other proprieties were added such as eating behaviour, proneness to injuries, sexual behavior, social differences, etc. Reports issued in 2005-2006 and 2009-2010 included more than 60 parameters divided into 4 basic groups: "social context" (family, peers, school); "health outcomes" (positive health, medically attended injuries, body weight); "health behaviours" (eating behaviour, oral health, physical activity and sedentary behaviour); "risk behaviours" (smoking, alcohol and cannabis use, sexual behaviour, fighting and bullying). In 2016 the last available report was published that contained results of the last questioning performed in 2013-2014 and included a number of new priorities, such as support by family and peers, most serious injury requiring medical treatment, migration, and cyberbulling.

Most results are systematized as per geographic regions (countries and regions in Europe and North America); age (11, 13 and 15); sex (boys or girls); social and economic status.

Our research goal was to analyze and systematize risk factors prevalence among Russian teenagers aged 11,13 and 15 taken in time dynamics.

\section{Data and methods}

We took data from international reports issued within "Health Behaviour in SchoolAged Children [HBSC]" project in 1993, 1997, 2001, 2005, 2009 and 2013, processed them statistically, performed comparative analysis and interpretation.

Research data were statistically processed with non-parametric analysis techniques. Data were accumulated, adjusted and systematized, and results visualized, with Microsoft Office Excel 2016. We performed statistical analysis with STATISTICA 13.3 package (developed by StatSoft Inc.) and Open Epi calculator (https://www.openepi.com).

Nominal and ordinal data were described with stating their $\%$ fractions.

Risk factors prevalence was compared between boys and girls; between 11-eyar and 15-year old teenagers; between different years; between different countries. 
We compared nominal data with Pearson's $\chi^{2}$ test that allowed assessing significance of discrepancies between actual number of outcomes or qualitative characteristics of a sampling that were included into each category, and a theoretical quantity that could be expected in examined groups, a zero hypothesis being valid. Results were considered to be statistically significant at $\mathrm{p} \leq 0.05$.

To test a hypothesis on a possible statistic correlation between risk factors and age (or years), we applied $\chi^{2}$ for a linear trend. In addition, we assessed whether an existing dependence could possibly deviate from a linear one. To do that, we deducted $\chi^{2}$ for a linear trend from overall $\chi^{2}$ value and compared an obtained difference with a critical $\chi^{2}$ value for remaining degrees of freedom. If a difference was smaller than critical $\chi^{2}$ value, it meant that data didn't deviate from a linear statistical correlation. To assess an amount of an effect, we applied Kramer V-test for nominal data (sex) (interpreted according to Rea \& Parker recommendations) $[19,20]$.

To compare parameters obtained for Russian schoolchildren with those obtained for their counterparts from other countries, we divided countries that took part in HBSC research into three equal groups: favorable, intermediate, or unfavorable situation depending on a factor. A situation (favorable, intermediate, or unfavorable) prevailing in the RF in comparison with other countries from the list was established for each factor.

\section{Results}

\section{Age-related peculiarities}

Drastic changes occur in endocrine glands functioning in teenagers aged $11-15$. It is a period when pubescence develops rapidly in girls and starts in boys. Usually there is a prepubertal jump in a teenager's growth combined with certain disharmonic changes as sexrelated physical and mental features occur and develop. It is the most difficult stage in psychological development when a person's will, consciousness and morality start to shape. Frequently it is a rather dramatic re-assessment of all life values, attitudes towards oneself, parents, contemporaries, and society in general. So, extreme judgments and behavior are quite possible as well as striving for self-assertion and conflicts [14, 21].

At this age teenagers usually adopt their behavioral patterns and attitudes towards their health; these attitudes in future will influence their health, welfare, and life quality. To improve teenagers' health and secure their welfare, it is necessary to obtain epidemiologic data on age-related differences in teenagers' perceptions of their social environment and on prevalence of both health-improving factors and factors that cause health risks.

Teenagers tended to perceive their educational environment in a more negative way as they grew; thus, 27.5\% 11-year old teenagers stated "they like school a lot", but their number dropped to $19.3 \%$ and $16.8 \%$ at 13 and 15 respectively (Table 1 ). Interaction and socializing between teenagers have changed considerably over recent years due to rapid development of social networks and other electronic mass media. A number of teenagers that every day communicated with their peers via electronic media communication (EMC) grew with age, from $46.8 \%$ to $53.3 \%$ respectively; it was revealed that every day $29.5 \% 11$-year old teenagers communicated with their friends in social media; they figure amounted to $42.5 \%$ among 13-year old teenagers, and to $51.0 \%$, among 15-year old ones; $21.0 \%, 32.0 \%$, and $41.5 \%$ teenagers respectively communicated with their friends via text messages.

Negative trends determined by age-related changes can be seen in a way teenagers estimated their health in a period from 11 to 15 : they started to think their health was poor more frequently $(20.0 \%$ and $27.5 \%$ accordingly); they had multiple health complaints (32.6\% and $35.4 \%$ accordingly). Number of teenagers who were satisfied with their life also dropped with age, from $82.0 \%$ among 11-year old, to $79.9 \%$, among 13 -year old, and to $78.4 \%$, among those who are 15. 11-year old boys and girls tended to have overweight and obesity more frequently than those who were $15(18.0 \%$ against $10.7 \%)$. However, it was 15 -year old teenagers who thought they were too fat more frequently than 11-year old ones $(15.9 \%$ against $14.6 \%)$ and who engaged in weight-reduction behaviour, $14.1 \%$ against 
Table 1

Age-related peculiarities of health-influencing behavioral factors among Russian teenagers aged 11,13 and 15

\begin{tabular}{|c|c|c|c|c|c|c|c|c|c|}
\hline \multirow{2}{*}{ Parameters } & \multicolumn{3}{|c|}{ Mean value $(\%)$} & \multirow{2}{*}{$\mathrm{N}$} & \multirow{2}{*}{$\begin{array}{l}\chi^{2} \\
\mathrm{~d}=2\end{array}$} & \multirow[b]{2}{*}{$\mathrm{p}$} & \multirow{2}{*}{$\begin{array}{c}\chi_{1 . t .}^{2} \\
d=1\end{array}$} & \multirow[b]{2}{*}{$\mathrm{p}$} & \multirow[t]{2}{*}{$\chi^{2}-\chi_{1 . t .}^{2}$} \\
\hline & 11 & 13 & 15 & & & & & & \\
\hline Liking school a lot & 27.5 & 19.3 & 16.8 & 29,562 & 448.9 & $<0.001$ & 437.5 & $<0.001$ & 11.4 \\
\hline Communicate daily & 46.8 & 51.3 & 53.3 & 21,237 & 62.8 & $<0.001$ & 52.8 & $<0.001$ & 10.0 \\
\hline social network & 29.5 & 42.5 & 51.0 & 4,274 & 123.7 & $<0.001$ & 121.0 & $<0.001$ & $2.7 * 1$ \\
\hline text messages & 21.0 & 32.0 & 41.5 & 4,274 & 125.0 & $<0.001$ & 124.7 & $<0.001$ & $0.3^{*}$ \\
\hline Fair or poor self-rated health & 20.0 & 23.5 & 27.5 & 25,511 & 173.3 & $<0.001$ & 173.1 & $<0.001$ & $0.2^{*}$ \\
\hline Multiple health complaints & 32.6 & 33.9 & 35.4 & 25,511 & 14.4 & $<0.001$ & 14.3 & $<0.001$ & $0.1 *$ \\
\hline High life satisfaction & 82.0 & 79.9 & 78.4 & 25,511 & 35.5 & $<0.001$ & 35.1 & $<0.001$ & $0.4 *$ \\
\hline Overweight & 18.0 & 12.5 & 10.7 & 17,680 & 146.8 & $<0.001$ & 135.9 & $<0.001$ & 10.9 \\
\hline Feeling too fat & 14.6 & 15.6 & 15.9 & 25,511 & 5.9 & $<0.001$ & 5.2 & $<0.001$ & $0.7^{*}$ \\
\hline $\begin{array}{l}\text { Engaged in weight-reduction } \\
\text { behaviour }\end{array}$ & 10.9 & 11.4 & 14.1 & 25,511 & 48.8 & $<0.001$ & 41.9 & $<0.001$ & 6.9 \\
\hline $\begin{array}{l}\text { Injuries required medical inter- } \\
\text { vention }\end{array}$ & 46.0 & 44.0 & 40.1 & 25,511 & 61.7 & $<0.001$ & 59.7 & $<0.001$ & $2.0^{*}$ \\
\hline Eating breakfast every day & 66.4 & 62.8 & 59.0 & 25,511 & 99.3 & $<0.001$ & 99.3 & $<0.001$ & $0.0^{*}$ \\
\hline Daily fruit consumption & 34.4 & 32.1 & 27.4 & 25,511 & 100.9 & $<0.001$ & 96.8 & $<0.001$ & 4.1 \\
\hline Daily vegetables consumption & 34.0 & 32.8 & 31.8 & 25,511 & 9.9 & $<0.001$ & 9.8 & $<0.001$ & $0.1^{*}$ \\
\hline 60 minutes of MVPA daily & 17.3 & 15.2 & 11.8 & 17,680 & 72.0 & $<0.001$ & 70.9 & $<0.001$ & $1.1^{*}$ \\
\hline $\begin{array}{l}\text { Using a computer for } 2 \text { or more } \\
\text { hours a day }\end{array}$ & 34.0 & 43.2 & 52.2 & 17,680 & 401.8 & $<0.001$ & 401.8 & $<0.001$ & $0.0^{*}$ \\
\hline Weekly smoking & 3.0 & 11.1 & 19.1 & 32,659 & 1448.4 & $<0.001$ & 1448.4 & $<0.001$ & $0.0^{*}$ \\
\hline Weekly alcohol consumption & 5.1 & 11.4 & 18.1 & 32,659 & 903.5 & $<0.001$ & 903.5 & $<0.001$ & $0.0^{*}$ \\
\hline $\begin{array}{l}\text { Having been drunk on } 2 \text { or } \\
\text { more occasions }\end{array}$ & 3.5 & 12.2 & 24.9 & 32,659 & 2178.2 & $<0.001$ & 2151.4 & $<0.001$ & $26.8^{*}$ \\
\hline $\begin{array}{l}\text { Fighting three or more times in } \\
\text { the last } 12 \text { months }\end{array}$ & 18.9 & 16.9 & 12.6 & 17,881 & 68.9 & $<0.001$ & 64.9 & $<0.001$ & 4.0 \\
\hline $\begin{array}{l}\text { Being a victim of bullying at } \\
\text { school }\end{array}$ & 23.0 & 18.8 & 11.3 & 17,881 & 222.1 & $<0.001$ & 214.2 & $<0.001$ & 7.9 \\
\hline
\end{tabular}

1 * значение разности меньше критического значения $\chi^{2}(3,841)$ для $\mathrm{d}=1$

$10.9 \%$ accordingly. Number of injuries that required medical intervention fell with age $(46.0 \%$ among 11 year-old teenagers, $44.0 \%$, among 13-year old, and $40.1 \%$, among 15 -year old ones).

Number of teenagers who ate breakfast, fruit and vegetables every day also tended to decrease with age while prevalence of low physical activity (less than 60 minutes of moderate-tovigorous physical activity (MVPA) per day), on the contrary grew and a share of teenagers who had daily physical activity also went down (17.3\% among 11-year old, $15.2 \%$, among 13 -year old, and $11.8 \%$, among 15 -year old teenagers). There was also a growth in number of teenagers who used a computer for 2 or more hours a day, $34.0 \%, 43.2 \%$, and $52.2 \%$ respectively.
Behavioral patterns that could cause health risk (such as smoking or alcohol use) prevailed greater among 15-year old school children against 11-year old ones. With age, more teenagers smoked daily $(3.0 \%$ among 11 -year old, $11.1 \%$, among 13-year old, and 198.1\% among 15-year old ones) and drank alcohol every day (5.1\%, $11.4 \%$, and $18.1 \%$ accordingly). Number of those teenagers who have been drunk on 2 or more occasions also grew with age $(3.5 \%$, $12.2 \%$, and $24.9 \%$ respectively).

Number of teenagers who have been involved in a physical fight at least three times in the last 12 months tended to decrease with age (18.9\%, 16.9\%, and $12.6 \%$ accordingly) as well as number of teenagers who have been bullied at school (23.0 \%, 18.8\%, and $11.3 \%$ accordingly). 


\section{Gender differences}

Gender differences are usually related to psychological traits and behavioral patterns that are formed within a culture on the basis of differences between sexes. Men and women tend to have their specific gender roles in a society. Culture makes for these or those differences in behavioral patterns between men and women becoming apparent as well as differences related to their social roles, responsibilities, and obligations. Gender inequality index introduced by the UN allows evaluating a correlation between gender inequality and health of population in various countries and regions. It reveals that population in countries where social inequality is higher tend to have poor health as regards both sexes; in other words, gender ine- quality causes damage to health of both young men and women [22].

To create efficient and targeted activities aimed at improving health and preventing diseases, it is necessary to have an insight into gender differences and similarities.

Girls had positive school experience more frequently than boys (Table 2) as they more often stated they "liked school a lot" (22.8\% against $19.6 \%$ ) and they had good or very good perceived school performance (56.3\% against 51.1\%). However, girls stated that they felt pressured by schoolwork more frequently than boys $(32.4 \%$ and $29.4 \%$ accordingly).

Girls communicated with their friends via EC, with text messages, or contacted them in social media more frequently than boys.

Table 2

Gender peculiarities of health-influencing behavioral factors among Russian teenagers aged 11,13 , and 15

\begin{tabular}{|c|c|c|c|c|c|c|c|}
\hline \multirow{2}{*}{ Parameters } & \multicolumn{2}{|c|}{ Mean value $(\%)$} & \multirow{2}{*}{$\mathrm{N}$} & \multirow{2}{*}{$\begin{array}{c}\chi^{2} \\
d=1\end{array}$} & & \multirow{2}{*}{ f-test } & \multirow{2}{*}{ Correlation } \\
\hline & boys & girls & & & & & \\
\hline Liking school a lot & 19.6 & 22.8 & 30,280 & 46.1 & $<0.001$ & 0.04 & insignificant \\
\hline $\begin{array}{l}\text { Good or very good perceived school } \\
\text { performance }\end{array}$ & 51.1 & 56.3 & 26,229 & 70.1 & $<0.001$ & 0.05 & insignificant \\
\hline Feeling pressured by schoolwork & 29.4 & 32.4 & 26,229 & 27.6 & $<0.001$ & 0.03 & nsignificant \\
\hline Communicate daily via EMC & 42.7 & 58.3 & 21,513 & 525.3 & $<0.001$ & 0.16 & \\
\hline socis & 35.3 & 46.7 & 4,716 & 55.1 & $<0.001$ & 0.11 & \\
\hline text mess & 24.3 & 38.7 & 4,716 & 108.8 & $<0.001$ & 0.15 & weak \\
\hline Fair or poor self-r & 18.4 & 28.9 & 26,229 & 73.3 & $<0.001$ & 0.05 & nsignificant \\
\hline Multip & 27.3 & 40.6 & 26,229 & 514.0 & $<0.001$ & 0.14 & \\
\hline High life sc & 82.1 & 78.8 & 26,229 & 46.2 & $<0.001$ & 0.04 & icant \\
\hline required medi & 49.1 & 37.7 & 26,229 & 346.2 & $<0.001$ & 0.12 & \\
\hline Overweight & 17.9 & 9.6 & 18,128 & 268.8 & $<0.001$ & 0.12 & weak \\
\hline Feeling too fat & 11.8 & 18.9 & 26,229 & 254.9 & $<0.001$ & 0.09 & insignificant \\
\hline Engaged in weight-reduction behaviour & 6.9 & 17.3 & 26,229 & 680.9 & $<0.001$ & 0.16 & weak \\
\hline Eating breakfast every day & 66.3 & 59.1 & 26,229 & 147.2 & $<0.001$ & 0.08 & insignificant \\
\hline Oral health & 47.0 & 64.9 & 34,339 & 1114.0 & $<0.001$ & 0.18 & weak \\
\hline 60 minutes of MVPA daily & 18.4 & 11.1 & 18,128 & 195.0 & $<0.001$ & 0.10 & \\
\hline $\begin{array}{l}\text { Playing games on a compute } \\
\text { console for } 2 \text { or more hours }\end{array}$ & 53.7 & 32.4 & 18,128 & 832.7 & $<0.001$ & 0.21 & weak \\
\hline Weekly smoking & 12.7 & 9.4 & 34,339 & 96.4 & $<0.001$ & 0.05 & insignificant \\
\hline Weekly alcohol consumption & 13.7 & 9.4 & 34,339 & 154.9 & $<0.001$ & 0.07 & insignificant \\
\hline $\begin{array}{l}\text { Having been drunk on } 2 \text { or more oc- } \\
\text { casions }\end{array}$ & 15. & 13.4 & 32 & 29.3 & $<0.001$ & 0.03 & cant \\
\hline $\begin{array}{l}\text { Fighting three or more times in the last } \\
12 \text { months }\end{array}$ & 25 & 7.3 & 26 & 1565.0 & $<0$ & 0.24 & weak \\
\hline Bulling others at school & 20.6 & 12.8 & 26,229 & 292.1 & $<0.001$ & 0.11 & weak \\
\hline
\end{tabular}


More girls than boys estimated their health as poor ( $28.9 \%$ against $18.4 \%$ accordingly); girls tended to complain about their health more frequently (40.6\% against $27.3 \%)$ and were less satisfied with their life $(78.8 \%$ against $82.1 \%$ accordingly).

Injuries that required medical intervention more frequently occurred among boys than girls (49.1\% against $37.7 \%$ respectively).

More boys than girls tended to have overweight or obesity $(17.9 \%$ against $9.6 \%$ accordingly) but girls stated they was too fat (18.9\% against $11.8 \%)$ and that they engaged in weight-reduction behaviour $(17.3 \%$ against $6.9 \%$ ) more frequently than boys.

A number of those who had breakfast every day was higher among boys than girls $66.3 \%$ against $59.1 \%$ ). Girls stated that they brushed their teeth regularly (more than once a day) more frequently than boys (64.9\% against $47.0 \%$ ).

Boys were more physically active than girls $(18.4 \%$ against $11.1 \%)$ but at the same time they much more frequently admitted playing games on a computer or games console for 2 or more hours a day ( $53.7 \%$ against $32.4 \%$ ).

There were distinctive gender differences among Russian teenagers as regards behavioral patterns that were hazardous for their health as boys were more prone to risky behavior than girls.

Smoking, alcohol use, and drunkenness were more widely spread among 15-year old boys. Over the last decade there has been some leveling in traditional gender differences and gender convergence as regards certain risky behavioral patterns among 11 and 13-year old boys and girls as more 11- and 13-year old girls started to smoke, alcohol or cannabis use.

Boys took active part in fights $(25.0 \%$ against 7.3\%) and have bulled other at school (20.6\% against $12.8 \%)$ more often than girls.

Changes in prevalence of health-influencing behavioral factors taken in dynamics over several years

Most researchers are more interested in data that allow making some judgments on changes that happen over tine in their regions and countries; data on such changes helps them assess impacts exerted by health-improving activities in their country or a region.

There were some positive trends observed in 2013-2014 in comparison with previous years (Table 3 ); thus, we detected a growth in number of teenagers who liked school $(15.5 \%$ in 2001 against $27.5 \%$ in 2013), who reported good or very good perceived school performance (32.2\% in 2005 against $28.0 \%$ in 2013); there was a decrease in number of children who thought their health was poor $(31.7 \%$ in 2001 against $17.0 \%$ in 2013) and who complained about their health $(37.0 \%$ in 2005 against 32.7 in 2013 ); only $76.3 \%$ were satisfied with their life in 2001 but in 2013 their number increased up to $82.2 \%$.

But still, we detected certain negative trends in 2013-2014 in comparison with 2005-2006. Thus, there was a growth in traumatism among 15-year old girls $(35.3 \%$ in 2005 against $39.7 \%$ in 2013); more teenagers had overweight and obesity (9.8\% in 2005 against $19.7 \%$ in 2013) and thought they were "too fat" (10.8\% in 2005 against $26.3 \%$ in 2013).

There are also some other positive trends as regards health behaviour such as an increase in number of schoolchildren who eat fruit every day $(26.8 \%$ in 2001 against $35.7 \%$ in 2013) and decrease in number of those who consumed soft drinks daily $(26.2 \%$ in 2005 against $10.8 \%$ in 2013) and who watched TV for 2 or more hours a day $(73.0 \%$ in 2005 against $60.0 \%$ in 2013 ).

In 2013-2014 fewer schoolchildren had breakfast on weekdays than in previous years $(61.2 \%$ in 2005 against $59.3 \%$ in 2013$)$ or brushed their teeth more than once a day $(63.7 \%$ in 1997 against $57.3 \%$ in 2013). There was a considerable growth in number of children who used a computer for 2 or more hours a day at a PC (19.8\% in 2005 against $57.7 \%$ in 2013).

We detected some positive trends as regards risky behavior; thus a number of children who smoked every week went down in 2013 in comparison with 2013 (14.7\% against $8.7 \%$ accordingly); fewer teenagers drank alcohol every week $(16.7 \%$ in 2005 against $6.2 \%$ in 2013); there was also a decrease in number of teenagers who had been drunk 2 or 
Table 3

Trends in health-influencing behavioral factors taken in dynamics over years among Russian teenagers aged 11,13 , and 15

\begin{tabular}{|c|c|c|c|c|c|c|c|c|c|c|c|c|c|c|}
\hline \multirow[b]{2}{*}{ Parameters } & \multicolumn{6}{|c|}{ Mean value (\%) } & \multirow[b]{2}{*}{$\mathrm{N}$} & \multirow[b]{2}{*}{$\chi^{2}$} & \multirow[b]{2}{*}{$\mathrm{d}$} & \multirow[b]{2}{*}{$\mathrm{p}$} & \multirow{2}{*}{$\chi_{\mathrm{d}=1}^{2}$} & \multirow[b]{2}{*}{$\mathrm{p}$} & \multirow{2}{*}{$\begin{array}{l}\chi^{2}- \\
\chi_{1 . t .}^{2}\end{array}$} & \multirow{2}{*}{$\begin{array}{l}\text { Linear } \\
\text { trend } \\
\text { revealed }\end{array}$} \\
\hline & 1993 & 1997 & 2001 & 2005 & 2009 & 2013 & & & & & & & & \\
\hline $\begin{array}{l}\text { Liking school } \\
\text { a lot }\end{array}$ & - & 19.3 & 15.5 & 19.3 & 24.0 & 27.5 & 30,274 & 306.5 & 3 & $<0.001$ & 306.0 & $<0.001$ & 0.5 & 2001-2013 \\
\hline $\begin{array}{l}\text { Feeling pressured } \\
\text { by schoolwork }\end{array}$ & - & - & 30.8 & 32.2 & 31.2 & 28.0 & 26,223 & 25.0 & 2 & $<0.001$ & 22.9 & $<0.001$ & 2.1 & 2005-2013 \\
\hline $\begin{array}{l}\text { Fair or poor } \\
\text { self-rated health }\end{array}$ & - & - & 31.7 & 26.3 & 19.7 & 17.0 & 26,223 & 438.7 & 3 & $<0.001$ & 429.9 & $<0.001$ & 8.8 & - \\
\hline $\begin{array}{l}\text { Multiple health } \\
\text { complaints }\end{array}$ & - & - & 31.7 & 37.0 & 34.5 & 32.7 & 26,223 & 26.0 & 2 & $<0.001$ & 25.8 & $<0.001$ & 0.2 & 2005-2013 \\
\hline $\begin{array}{l}\text { High life satis- } \\
\text { faction }\end{array}$ & - & - & 76.3 & 79.5 & 82.3 & 82.2 & 26,223 & 70.5 & 2 & $<0.001$ & 70.4 & $<0.001$ & 0.1 & 2001-2009 \\
\hline Overweight & $\begin{array}{lll}- & \\
\end{array}$ & $\begin{array}{lll}- \\
\end{array}$ & $\begin{array}{llll}- & & & \end{array}$ & 9.8 & 11.7 & 19.7 & 8,122 & 267.4 & 2 & $<0.001$ & 237.2 & $<0.001$ & 30.2 & $\begin{array}{llll}- & \\
\end{array}$ \\
\hline Feeling too fat & $\begin{array}{lll}- & \\
\end{array}$ & - & 9.3 & 10.8 & 15.0 & 26.3 & 6,223 & 816.4 & 3 & $<0.001$ & 692.7 & $<0.001$ & 123.7 & - \\
\hline $\begin{array}{l}\text { Injuries required } \\
\text { medical interven- } \\
\text { tion*2 }\end{array}$ & - & - & 36.3 & 35.3 & 39.3 & 39.7 & 13,902 & 17.6 & 2 & $<0.001$ & 15.0 & $<0.001$ & 2.6 & 2005-2013 \\
\hline $\begin{array}{l}\text { Eating breakfast } \\
\text { every day }\end{array}$ & - & - & 68.7 & 61.2 & 61.7 & 59.3 & 223 & 4.5 & 3 & $<0.001$ & 111.8 & 0.001 & 2.7 & - \\
\hline $\begin{array}{l}\text { Daily fruit con- } \\
\text { sumption }\end{array}$ & - & - & 26.8 & 29.3 & 33.3 & 35.7 & 26,223 & 35.6 & 3 & $<0.001$ & 134.1 & $<0.001$ & 1.5 & 2001-2013 \\
\hline $\begin{array}{l}\text { Daily consump- } \\
\text { tion of soft drink }\end{array}$ & - & - & 21.7 & 26.2 & 23.0 & 10.8 & 26,223 & 433.7 & 2 & $<0.001$ & 390.0 & $<0.001$ & 43.7 & - \\
\hline Oral health & 38.3 & 63.7 & 61.3 & 60.7 & 57.3 & 54.3 & 33 & 87.4 & 4 & 01 & 101.1 & $<0.001$ & 3 & 1997-2013 \\
\hline $\begin{array}{l}\text { Watching TV for } \\
2 \text { or more hours } \\
\text { a day }\end{array}$ & - & - & - & 73.0 & 67.0 & 60.0 & 18,122 & 34.7 & 2 & $<0.001$ & 234.3 & $<0.001$ & 0.4 & 2005-2013 \\
\hline $\begin{array}{l}\text { Using a computer } \\
\text { for } 2 \text { or more } \\
\text { hours a day }\end{array}$ & - & - & - & 19.8 & 51.8 & 57.7 & 18,122 & 2,324.1 & 2 & $<0.001$ & $2,063.6$ & $<0.001$ & 260.5 & - \\
\hline Weekly smoking & 10.1 & 11.4 & 12.4 & 14.7 & 9.2 & 8.7 & 34,333 & $\begin{array}{c}61.2 \\
145.4\end{array}$ & \begin{tabular}{|l|}
3 \\
2 \\
\end{tabular} & $\begin{array}{l}<0.001 \\
<0.001\end{array}$ & \begin{tabular}{|c|}
58.9 \\
123.1 \\
\end{tabular} & $\begin{array}{l}<0.001 \\
<0.001\end{array}$ & \begin{tabular}{|c|}
2.3 \\
22.3 \\
\end{tabular} & $\begin{array}{l}1993-2005- \\
2005-2013 \\
\end{array}$ \\
\hline $\begin{array}{l}\text { Weekly alcohol } \\
\text { consumption }\end{array}$ & 9.8 & 13.5 & 14.7 & 16.7 & 8.3 & 6.2 & 34,333 & $\begin{array}{l}106.5 \\
396.9 \\
\end{array}$ & $\begin{array}{l}3 \\
2 \\
\end{array}$ & $\begin{array}{l}<0.001 \\
<0.001 \\
\end{array}$ & \begin{tabular}{|l|}
102.1 \\
363.2 \\
\end{tabular} & $\begin{array}{l}<0.001 \\
<0.001 \\
\end{array}$ & \begin{tabular}{|c|}
4.3 \\
33.7 \\
\end{tabular} & $\begin{array}{c}1993-2005- \\
2005-2013 \\
\end{array}$ \\
\hline $\begin{array}{l}\text { Having been } \\
\text { drunk on } 2 \text { or } \\
\text { more occasions }\end{array}$ & 8.0 & 13.3 & 19.3 & 20.5 & 13.3 & 6.7 & 34,333 & $\begin{array}{l}376.4 \\
467.7\end{array}$ & $\begin{array}{l}3 \\
2\end{array}$ & $\begin{array}{l}<0.001 \\
<0.001\end{array}$ & $\begin{array}{l}345.2 \\
467.5\end{array}$ & $\begin{array}{l}<0.001 \\
<0.001\end{array}$ & \begin{tabular}{|c|}
31.2 \\
0.2
\end{tabular} & $\begin{array}{c}-1993-2005 \\
2005-2013\end{array}$ \\
\hline $\begin{array}{l}\text { Fighting three or } \\
\text { more times in the } \\
\text { last } 12 \text { months }\end{array}$ & - & - & 18.3 & 18.3 & 15.2 & 12.7 & 26,223 & 75.2 & 2 & $<0.001$ & 74.9 & $<0.001$ & 0.3 & 2005-2013 \\
\hline $\begin{array}{l}\text { Being a victim of } \\
\text { bullying at school }\end{array}$ & - & - & 17.7 & 16.3 & 17.8 & 18.8 & 26,223 & 13.8 & 2 & $<0.001$ & 13.7 & $<0.001$ & 0.1 & 2005-2013 \\
\hline $\begin{array}{l}\text { Bulling others at } \\
\text { school } *^{2}\end{array}$ & - & - & 11.3 & 13.0 & 13.0 & 13.3 & 13,902 & 8.6 & 3 & 0.03 & 6.1 & 0.013 & 2.5 & $2001-2013$ \\
\hline
\end{tabular}

${ }^{2}$ Data are given for girls. 
more times in their life $(20.5 \%$ in 2005 against $6.7 \%$ in 2013 ) and in a number of fights with teenagers' participation $(18.3 \%$ in 2005 against $12.7 \%$ in 2013).

But still there were some negative trends in 2013-2014; thus there was a increase in number of teenagers who have been bullied at school (16.3\% in 2005 against $18.8 \%$ in 2013) and in number of girls who have bullied other children at school $(11.3 \%$ in 2001 against $13.3 \%$ in 2013).

Differences in parameters revealed in the $R F$ and other countries

There are always differences in health and their social determinants revealed between different countries. Since HBSC was first introduced, it has become quite possible to reveal peculiarities related to health and lifestyle within a context determined by political and economical changes. Health parameters detected for children in the RF are worse than those detected for children in other countries.
Russian children aged 11 and 13 stated they liked school or thought their school performance were good less frequently than their peers in other countries; most teenagers aged 11, 13, and 15 didn't think their classmates were kind and helpful; they preferred to communicate with their friends via EMC more frequently than personally (table 4).

Russian schoolchildren more frequently thought their health was poor and were less satisfied with their life; injuries required medical intervention prevailed among 11- and 15year-old teenagers. However, Russian teenagers suffered from overweight and obesity less frequently than their foreign peers; they also didn't think they were "too fat" and engaged in weight-reduction behaviour as often as schoolchildren in other countries.

Fewer Russian teenagers ate fruit every day (11- and 13-year-old ones), brushed their teeth more than once a day (11- and 15-year-old ones), or had sufficient physical activity

Table 4

Situation in the RF in comparison with other countries that take part in HBSC

\begin{tabular}{|l|c|c|}
\hline \multicolumn{1}{|c|}{ Parameter } & $\begin{array}{c}\text { Situation in the RF against } \\
\text { other countries }\end{array}$ & Age groups or years \\
\hline Liking school a lot & unfavorable & 11,13 \\
\hline Good or very good perceived school performance & unfavorable & 11,13 \\
\hline Classmates are kind and helpful & unfavorable & $11-15$ \\
\hline Communicate daily via EMC & unfavorable & $11-15$ \\
\hline Fair or poor self-rated health & unfavorable & $11-15$ \\
\hline High life satisfaction & unfavorable & $11-15$ \\
\hline Injuries required medical intervention & unfavorable & 11,15 \\
\hline Overweight & favorable & 13,15 \\
\hline Feeling too fat & favorable & $11-15$ \\
\hline Engaged in weight-reduction behaviour & intermediate & $11-15$ \\
\hline Eating breakfast every day & intermediate & $11-15$ \\
\hline Daily fruit consumption & unfavorable & 11,13 \\
\hline Daily sweets consumption & unfavorable & $2013 ; 11,13$ \\
\hline Daily consumption of soft drink & favorable & $2013 ; 11,13$ \\
\hline Oral health & unfavorable & $2009-2013 ; 11-15$ \\
\hline 60 minutes of MVPA daily & unfavorable & 11 \\
\hline Using a computer for 2 or more hours a day & unfavorable & $2013 ; 11-15$ \\
\hline $\begin{array}{l}\text { Playing games on a computer or games console for } \\
\text { 2 or more hours a day }\end{array}$ & unfavorable & 11 \\
\hline Weekly smoking & unfavorable & 11,13 \\
\hline Weekly alcohol consumption (beer, wine, or alcopops) & unfavorable & 13 \\
\hline Having been drunk on 2 or more occasions & favorable & 15 \\
\hline Fighting three or more times in the last 12 months & unfavorable & $11-15$ \\
\hline Being a victim of bullying at school & unfavorable & $11-15$ \\
\hline Bulling others at school & unfavorable & $11-15$ \\
\hline
\end{tabular}


(11-year-old ones) than their foreign peers. They also ate sweets (11- and 13-year-old teenagers) and used a computer and watched TV more often than schoolchildren in other countries.

Risky behavior patterns were more widely spread among younger Russian teenagers; thus 11-and 13-year-old teenagers smoked more than their foreign peers; 13-year-old ones use alcohol (beer, wine, or alcopops) more frequently. Russian schoolchildren were much more aggressive than their peers from European or North American countries as it was confirmed by greater prevalence of fights and bulling among Russian teenagers.

\section{Conclusions}

Any activities aimed at improving health and providing welfare of teenage boys and girls should be developed taking into account differences between age groups. Greater efforts should be made to create stimuli to pursue healthy lifestyle among younger teenagers. A school is the most relevant place to implement such activates as it allows combining knowledge and skills of teachers and medical personnel $^{3}[23,24]$.

Detected differences indicate that healthimproving and preventing strategies should be developed separately for boys and girls. A lot of risky behavioral patterns are more widely spread among boys; therefore, preventive activities should be more focused on them. It is necessary to be very attentive to any potential increase in risky behavioral patterns among girls that can be caused by gender convergence.

Over the last decade some positive trends have been revealed as regards prevalence of behavioral risk factors; however, when compared with their peers from other countries, Russian teenagers have more negative attitudes towards their school environment, more frequently think their health is poor, they consume less fruit, pay less attention to oral health, and are not so physically active. Risky behavioral patterns (smoking, alcohol use, and aggression) are more widely spread among younger Russian teenagers than their foreign peers.

When creating programs aimed at stimulating healthy lifestyle, one should take into account age and gender differences, as well as trends related to behavioral risk factors that can change over time and that can be objectively assessed with "Health Behaviour in School-Aged Children" questionnaire.

Funding. The research was not granted any sponsor support.

Conflict of interests. The authors state there is no any conflict of interests.

\section{References}

1. Baranov A.A. Sostoyanie zdorov'ya detei Rossiiskoi Federatsii [Children health in the Russian Federation]. Pediatriya zhurnal im. G.N. Speranskogo, 2012, vol. 91, no. 3, pp. 9-14 (in Russian).

2. Al'bitskii V.Yu. Zabolevaemost' detei i invalidnost' [Incidence and disability among children]. Rossiiskii pediatricheskii zhurnal, 2014, no. 10, pp. 32-35 (in Russian).

3. Goncharova O.V., Sokolovskaya T.A. Morbidity among children aged 0-14 years in the Russian Federation: longitudinal and prospective studies. Meditsinskii sovet, 2014, no. 6, pp. 6-8 (in Russian).

4. Gudinova Zh.V., Zhernakova G.N., Bolotova S.S., Gegechkori I.V. Assessment of the quality of information on the health of children in Russia: interregional comparisons and classification. Gigiena $i$ sanitariya, 2015, vol. 94, no. 3, pp. 77-82 (in Russian).

5. Kuchma V.R., Sukhareva L.M., Rapoport I.K., Shubochkina E.I., Skoblina N.A., Milushkina O.Yu. Population health of children, risks to health and sanitary and epidemiological wellbeing of students: problems, ways of solution and technology of the activity. Gigiena i sanitariya, 2017, vol. 96, no. 10, pp. 990-995 (in Russian).

6. Cattaneo A., Cogoy L., Macaluso A., Tamburlini G. Child health in the European Union. Luxemburg, European Commission Publ., 2012, 134 p.

\footnotetext{
${ }^{3}$ Harmonizing European and Russian approaches to theory and practice related to assessing quality of medical support provided for children at educational establishments. A concept for assessing quality of medical supports for schoolchildren: Guide / edited by V.R. Kuchma. - M.: RF Public Healthcare Ministry, Scientific Medical Center for Children's Health, 2016. - 85 p.
} 
7. Potrebny T., Wiium N., Haugstvedt A., Sollesnes R., Torsheim T., Wold B., Thuen, F. Health complaints among adolescents in Norway: A twenty-year perspective on trends. Available at: https://journals.plos.org/plosone/article?id=10.1371/journal.pone.0210509 (21.02.2019).

8. Kuindzhi N.N., Zorina I.G. Experience of application of social hygienic monitoring in hygiene of children and adolescents and the lessons resulting from it. Gigiena i sanitariya, 2012, vol. 91, no. 4, pp. 53-57 (in Russian).

9. Health 2020: a European policy framework supporting action across government and society for health and well-being.Copenhagen, WHO Regional Office for Europe, 2013. Available at: http:/www.euro. who.int/_ data/assets/pdf_file/0006/199536/Health2020-Short.pdf?ua=1 (14.04.2019).

10. Milushkina O.Y. $\mathrm{u}$. Physical Development and Lifestyle of Today's Schoolchildren. Vestnik Rossiiskogo gosudarstvennogo meditsinskogo universiteta, 2013, no. 3, pp. 68-71 (in Russian).

11. Valeeva E.R., Akberova G.R., Kladov D.Yu., Ziyatdinov A.I. Lifestyle of the students of specialized educational institutions and lyceum. Gigiena i sanitariya, 2014, vol. 93, no. 4, pp. 93-95 (in Russian).

12. Baranov A.A., Kuchma V.R., Namazova-Baranova L.S., Sukhareva L.M., Rapoport I.K. [et al]. Strategiya «Zdorov'e i razvitie podrostkov Rossii» (garmonizatsiya evropeiskikh i rossiiskikh podkhodov $\mathrm{k}$ teorii i praktike okhrany i ukrepleniya zdorov'ya podrostkov): Monografiya ["Health and development of teenagers in Russia (strategy)" (harmonizing European and Russian approaches to theoretical and practical aspects related to protecting and improving teenagers' health): a monograph]. Moscow, Izdatel'stvo «Pediatr» Publ., 2014, 112 p. (in Russian).

13. WHO Regional Office for Europe. Health for the World's Adolescents. A second chance in the second decade. Copenhagen, WHO Regional Office for Europe, 2012, 36 p.

14. Kuchma V.R., Sokolova S.B. Povedencheskie riski, opasnye dlya zdorov'ya shkol'nikov XXI veka [Behavioral health risks for schoolchildren in the 21st century: a monograph]. Moscow, FGAU «NMIC zdorov'ya detey» Minzdrava Rossii Publ., 2017, 170 p. (in Russian).

15. Quon E.C., McGrath J.J. Community, family, and subjective socioeconomic status: Relative status and adolescent health. Health Psychol, 2015, vol. 34, no. 6, pp. 591-601.

16. Ottova-Jordan V., Smith O.R., Augustine L., Gobina I., Rathmann K., Torsheim T. [et al]. Trends in health complaints from 2002 to 2010 in 34 countries and their association with health behaviours and social context factors at individual and macro-level. Eur. J. Pub. Health, 2015, no. 25, pp. 83-89.

17. Kim H.H.S., Chun J. Analyzing multilevel factors underlying adolescent smoking behaviors: the roles of friendship network, family relations, and school environment. Journal of School Health, 2018, vol. 88, no. 6, pp. 434-443. DOI: $10.1111 /$ josh. 12630

18. Health Behaviour in School-aged Children (HBSC) study: international report from the 2013/2014 survey. World Health Organization. Available at: http://www.euro.who.int/en/health-topics/Life-stages/child-and-adolescent-health/health-behaviour-in-school-aged-children-hbsc/hbsc-international-reports/ growing-up-unequal.-hbsc-2016-study-20132014-survey (14.04.2019).

19. Grjibovski A.M. Analysis of nominal data (independent observations). Ekologiya cheloveka, 2008, no. 6, pp. 58-68 (in Russian).

20. Grjibovski A.M. Analysis of ordinal data. Ekologiya cheloveka, 2008, no. 8, pp. 56-62 (in Russian).

21. Viner R.M., Ozer E.M., Denny S., Marmot M., Resnick M., Fatusi A. [et al]. Adolescence and the social determinants of health. Lancet, 2012, vol. 9826, no. 379, pp. 1641-1652.

22. Költo A., Young H., Burke L., Moreau N., Cosma A., Magnusson J. [et al]. Love and Dating Patterns for Same-and Both-Gender Attracted Adolescents Across Europe. Journal of Research on Adolescence, 2018, vol. 28, pp. 772-778. DOI:10.1111/jora.12394

23. Eccles J.S., Roeser R.W. Schools as developmental contexts during adolescence. J. Res. Adolesc, 2011, vol. 21, no. 1, pp. 225-241.

24. Högberg B., Strandh M., PetersennS., Johansson K. Education system stratification and health complaints among school-aged children.Social Science \& Medicine, 2018, vol. 220, pp. 159-166. DOI: 10.1016/j.socscimed.2018.11.007

Kuchma V.R., Sokolova S.B. Basic trends in behavioral health risks. Health Risk Analysis, 2019, no. 2, pp. 4-13. DOI: 10.21668/health.risk/2019.2.01.eng

Received: 07.05.2019

Accepted: 30.05 .2019

Published: 30.06 .2019 